\title{
Bi Incorporation and Segregation in the MBE-Grown GaAs-(Ga,Al)As-Ga(As,Bi) Core-Shell Nanowires
}

Janusz Sadowski ( $\square$ janusz.sadowski@lnu.se )

Polish Academy of Sciences

\section{Anna Kaleta}

Polish Academy of Sciences

\section{Serhii Kryvyi}

Polish Academy of Sciences

\section{Dorota Janaszko}

Polish Academy of Sciences

\section{Bogusława Kurowska}

Polish Academy of Sciences

\section{Marta Bilska}

Polish Academy of Sciences

\section{Tomasz Wojciechowski}

Polish Academy of Sciences

Jarosław Z. Domagala

Polish Academy of Sciences

Ana M. Sanchez

University of Warwick

Sławomir Kret

Polish Academy of Sciences

\section{Research Article}

Keywords: Nanowires, GaAs-(Ga,Al)As-Ga(As,Bi)

Posted Date: October 22nd, 2021

DOI: https://doi.org/10.21203/rs.3.rs-985481/v1

License: (9) This work is licensed under a Creative Commons Attribution 4.0 International License.

Read Full License 
Version of Record: A version of this preprint was published at Scientific Reports on April 9th, 2022. See the published version at https://doi.org/10.1038/s41598-022-09847-w. 


\section{Bi Incorporation and segregation in the MBE-grown}

\section{GaAs-(Ga,Al)As-Ga(As,Bi) core-shell nanowires}

Janusz Sadowski ${ }^{l, 2,}{ }^{*}$, Anna Kaleta ${ }^{I}$, Serhii Kryvyi ${ }^{l}$, Dorota Janaszko ${ }^{l}$, Bogustawa Kurowska ${ }^{l}$, Marta Bilska ${ }^{1}$, Tomasz Wojciechowski ${ }^{1,3}$, Jarostaw Z. Domagala ${ }^{1}$, Ana M. Sanchez ${ }^{4}$, and Stawomir Kret ${ }^{1, *}$

${ }^{1}$ Institute of Physics Polish Academy of Sciences, Aleja Lotnikow 32/46, PL-02668 Warsaw, Poland

${ }^{2}$ Department of Physics and Electrical Engineering, Linnaeus University, SE-39182 Kalmar, Sweden

${ }^{3}$ International Research Centre MagTop, Institute of Physics, Polish Academy of Sciences, Aleja Lotnikow 32/46, PL-02668 Warsaw, Poland

${ }^{4}$ Department of Physics, University of Warwick, Coventry CV4 7AL, United Kingdom corresponding authors:

janusz.sadowski@1nu.se

kret@ifpan.edu.pl 
Abstract. Incorporation of $\mathrm{Bi}$ into GaAs-(Ga,Al)As-Ga(As,Bi) core-shell nanowires grown by molecular beam epitaxy is studied with transmission electron microscopy. Nanowires are grown on GaAs(100) substrates with Au-droplet assisted mode. Bi-doped shells are grown at low temperature $\left(300{ }^{\circ} \mathrm{C}\right)$ with a close to stoichiometric $\mathrm{Ga} /$ As flux ratio. At low Bi fluxes, the $\mathrm{Ga}(\mathrm{As}, \mathrm{Bi})$ shells are smooth, with Bi completely incorporated into the shells. Higher Bi fluxes (Bi/As flux ratio $\sim 4 \%$ ) led to partial segregation of $\mathrm{Bi}$ as droplets on the nanowires sidewalls, preferentially located at the nanowire segments with wurtzite structure. We demonstrate that such Bi droplets on the sidewalls act as catalysts for the growth of branches perpendicular to the GaAs trunks. Due to the tunability between zinc-blende and wurtzite polytypes by changing the nanowire growth conditions, this effect enables fabrication of branched nanowire architectures with branches generated from selected (wurtzite) nanowire segments. 
Crystallization of $\mathrm{Ga}(\mathrm{As}, \mathrm{Bi})$ ternary alloy is challenging since $\mathrm{Bi}$ acts as a surfactant during the epitaxial growth of III-V semiconductor layers ${ }^{1}$. However application of dedicated thin film growth procedures allowed to obtain $\mathrm{Ga}(\mathrm{As}, \mathrm{Bi})$ solid solutions with percentage range $\mathrm{Bi}$ content. Partial replacement of As anions by Bi heavy element leads to a substantial band-gap reduction and enhanced spin-orbit splitting in $\mathrm{Ga}(\mathrm{As}, \mathrm{Bi})$, as compared with the binary GaAs host ${ }^{2}$. $\mathrm{Ga}(\mathrm{As}, \mathrm{Bi})$ solid solution was first obtained by metalorganic vapor phase epitaxy (MOVPE) ${ }^{3}$, and then by molecular beam epitaxy (MBE) ${ }^{4}$. The latter became the most conventional method of growing $\mathrm{Ga}(\mathrm{As}, \mathrm{Bi})$ thin films, since higher Bi contents were obtained than those achieved with use of the MOVPE technique ${ }^{5}$ GaBi binary compound does not occur in the crystalline form, ${ }^{6}$ and therefore the amount of $\mathrm{Bi}$ atoms residing in the As lattice sites in the GaAs crystal is limited. The highest $\mathrm{Bi}$ concentration in $\mathrm{Ga}(\mathrm{As}, \mathrm{Bi})$ reported so far is about $20 \%$, obtained in thin layers grown by MBE at optimized conditions. A significant energy gap reduction, down to 0.5 $\mathrm{eV}$, has been reported for $17.8 \% \mathrm{Bi}_{\text {content }}{ }^{7}$. Hence $\mathrm{Ga}(\mathrm{As}, \mathrm{Bi})$ bandgap can be tuned between that of binary GaAs (1.43 eV at room temperature) and $0.5 \mathrm{eV}$, making this material suitable for infrared optoelectronic applications ${ }^{8}$. Moreover, Ga(As,Bi) and other heavily Bi doped III-V semiconductors (e.g. antimonides) are expected to exhibit topological insulator properties ${ }^{9}$. These topological properties were first based on theoretical modelling, but they have recently been experimentally demonstrated for InBi binary compound hosting topologically protected surface states ${ }^{10,11}$. There is no similar experimental evidence of topological protection in $\mathrm{Ga}(\mathrm{As}, \mathrm{Bi})$ yet, but this ternary alloy has not been investigated in this context so far. Recently topological properties of Bi-alloyed III-V semiconductors (arsenides and antimonides) in the wurtzite (WZ) polytype have also been theoretically predicted ${ }^{12}$. Since WZ III-V compounds can easily be obtained in the quasi $1 \mathrm{D}(\mathrm{NW})$ geometry $^{13}$, the efforts to grow III-(V,Bi) ternary 
alloy NWs are interesting also in the context of topological materials. Although, $\mathrm{Ga}(\mathrm{As}, \mathrm{Bi})$ planar layers grown by MBE have been investigated for over two decades, there is very limited number of reports on $\mathrm{Ga}(\mathrm{As}, \mathrm{Bi})$ in the $\mathrm{NW}$ geometry. The main reason is quite extreme $\mathrm{MBE}$ growth conditions of $\mathrm{Ga}(\mathrm{As}, \mathrm{Bi})$ (in comparison with $\mathrm{GaAs})$; i.e., low growth temperature ( 200 $-350^{\circ} \mathrm{C}$ ) and close-to stoichiometric V/III elements flux ratio to avoid Bi surface segregation and to induce its substantial incorporation at As sites in the GaAs host lattice ${ }^{14,15}$. These growth conditions deviate from the GaAs NWs growth requirements, where higher substrate temperatures $\left(>500{ }^{\circ} \mathrm{C}\right)$ and high As excess are indispensable ${ }^{16}$. This implies that $\mathrm{Ga}(\mathrm{As}, \mathrm{Bi})$ NWs with significant Bi content can only be obtained as low-temperature shells grown on Bifree core NWs. This is similar to NWs implementing (Ga,Mn)As dilute ferromagnetic semiconductor with akin growth requirements, demanding even lower growth temperatures [ $100{ }^{\circ} \mathrm{C}$ lower than $\left.\mathrm{Ga}(\mathrm{As}, \mathrm{Bi})\right]$ and the same stoichiometric III/V flux ratio ${ }^{17,18,19,20}$. Recently the mixed phase WZ-ZB GaAs NWs exposed ex-situ to Bi vapor were studied by scanning tunneling microscopy ${ }^{21}$ but this study has little reference to our investigations of in-situ Bi incorporation.

In this paper we thoroughly investigate the radial distribution of Bi in GaAs NWs (not studied so far to our knowledge) and elucidate the emergence of side Bi droplets and branches on the wurtzite segments of GaAs NWs with $\mathrm{Ga}(\mathrm{As}, \mathrm{Bi})$ shells.

\section{Samples and Experimental Methods}

The core-shell nanowires were grown in a dedicated III-V MBE system. First the GaAs NW trunks were crystallized on GaAs(111)B substrates by the Au-assisted growth mode. $5 \AA$ thick gold film was deposited on epi-ready GaAs(111)B wafers in another MBE system, and transferred (in air) to the III-V one. The growth was monitored by reflection high energy electron 
diffraction (RHEED) system. The substrate temperature was controlled by the MBE substrate manipulator thermocouple, calibrated using $\operatorname{GaAs}(100)$ surface reconstruction transition temperatures $^{22}$. For that a piece of GaAs(100) wafer was placed in the vicinity of Au-coated GaAs(111)B dedicated to the NWs growth. Planar Ga(As,Bi) layer grown on GaAs(100) during the Ga(As,Bi) NW shells deposition serves also as references to compare Bi incorporation into ZB and WZ GaAs phase of planar layers and NW shells, respectively (see the Supplementary information). After preheating the substrates to $600{ }^{\circ} \mathrm{C}$ in the $\mathrm{MBE}$ growth chamber resulting in the thermal desorption of native oxide and formation of AuGa eutectic droplets at random surface sites of $\mathrm{GaAs}(111) \mathrm{B}$ wafer, the substrate temperature was decreased to $540{ }^{\circ} \mathrm{C}$ and $\mathrm{GaAs}$ NWs have been grown for 1-3 hours, depending on the sample. The Ga flux intensity (calibrated through a test growth on GaAs(100) substrate by RHEED intensity oscillations) corresponded to the planar growth rate of $0.2 \mu \mathrm{m} / \mathrm{h}$, which resulted in the axial NWs growth rate of about $1 \mu \mathrm{m} / \mathrm{h}$. As the source of arsenic the valved cracker cell has been used with cracking zone temperature of $950{ }^{\circ} \mathrm{C}$ i.e., As dimers were prevailing in the As flux. The As/Ga flux ratio during this growth stage was about 10. The growth of GaAs NW trunks was completed by closing Ga and As shutters. The latter was closed one minute after the first one. After closing the Ga shutter the substrate temperature was decreased for deposition of the NW shells. In the case of one sample (sample 3) prior to the $\mathrm{Ga}(\mathrm{As}, \mathrm{Bi})$ growth first about $30 \mathrm{~nm}(\mathrm{Ga}, \mathrm{Al}) \mathrm{As}$ shells have been grown at the substrate temperature of $400{ }^{\circ} \mathrm{C}$, with As and Ga flux ratios the same as used previously for the axial GaAs NWs growth. For the deposition of $\mathrm{Ga}(\mathrm{As}, \mathrm{Bi})$ shells the substrate temperature was further decreased to about $300{ }^{\circ} \mathrm{C}$. $\mathrm{Ga}(\mathrm{As}, \mathrm{Bi})$ shells have been deposited in close-tostoichiometric growth conditions i.e. with V/III flux intensity ratio close to 1. 
We investigate three different types of GaAs-Ga(As,Bi) NW samples. The Ga(As,Bi) shells were grown in similar conditions for all the samples, i.e. at the same substrate temperature (300 ${ }^{\circ} \mathrm{C}$ ) and As/Ga flux ratio ( 1). The main difference between the growths was the Bi flux intensity during deposition of the low temperature (LT) Ga(As,Bi) shells. Bi flux was generated from a standard Knudsen effusion cell. To obtain different $\mathrm{Bi}$ concentrations the $\mathrm{Bi}$ cell temperature was set to $540{ }^{\circ} \mathrm{C}$ or $580{ }^{\circ} \mathrm{C}$, corresponding to low $(1 \%)$ or high (2-4\%) Bi content in $\mathrm{Ga}(\mathrm{As}, \mathrm{Bi})$ shells of sample 1, (2 and 3), respectively. In each case the $\mathrm{Ga}(\mathrm{As}, \mathrm{Bi})$ shell was finished by the deposition of 4-7 nm thick LT GaAs. The detailed parameters of the samples are listed in Tab.1.

\begin{tabular}{|c|c|c|c|c|c|c|}
\hline Sample No & $\begin{array}{c}\text { GaAs core } \\
\text { NW length } \\
\text { (average) }\end{array}$ & $\begin{array}{c}\text { (Ga,Al)As } \\
\text { shell } \\
\text { thickness }\end{array}$ & $\begin{array}{c}\text { GaAs } \\
\text { shell } \\
\text { thickness }\end{array}$ & $\begin{array}{c}\text { Ga(As,Bi) } \\
\text { shell } \\
\text { thickness }\end{array}$ & $\mathbf{T}_{\mathbf{B i}}$ & $\begin{array}{c}\text { LT GaAs } \\
\text { shell } \\
\text { thickness }\end{array}$ \\
\hline $\mathbf{1}$ & $2 \mu \mathrm{m}$ & 0 & 0 & $22 \mathrm{~nm}$ & $540^{\circ} \mathrm{C}$ & $7 \mathrm{~nm}$ \\
\hline $\mathbf{2}$ & $4.5 \mu \mathrm{m}$ & 0 & 0 & $14 \mathrm{~nm}$ & $580^{\circ} \mathrm{C}$ & $4 \mathrm{~nm}$ \\
\hline $\mathbf{3}$ & $3.5 \mu \mathrm{m}$ & $14 \mathrm{~nm}$ & $0.3 \mathrm{~nm}$ & $26 \mathrm{~nm}$ & $580^{\circ} \mathrm{C}$ & $5 \mathrm{~nm}$ \\
\hline
\end{tabular}

Table 1. Parameters of the NW samples

Figure 1 shows the scanning electron microscopy (SEM) images of samples 1, 2 and 3 grown with low (sample 1) and high Bi flux (samples 2, and 3), at the Bi effusion cell temperature $\left(\mathrm{T}_{\mathrm{Bi}}\right)$ of $540{ }^{\circ} \mathrm{C}$ and $580{ }^{\circ} \mathrm{C}$, respectively. NW sidewalls in the sample 1 are smooth with no side droplets. The conical features at the NW tips (with clearly visible Au droplet at the very top) are due to the residual axial growth during the $\mathrm{Ga}(\mathrm{As}, \mathrm{Bi})$ shell deposition at $300{ }^{\circ} \mathrm{C}$. In samples 2 and 3 grown with $\mathrm{T}_{\mathrm{Bi}}=580{ }^{\circ} \mathrm{C}$ most of the NWs have side droplets, and short branches perpendicular to the main NW trunks. 

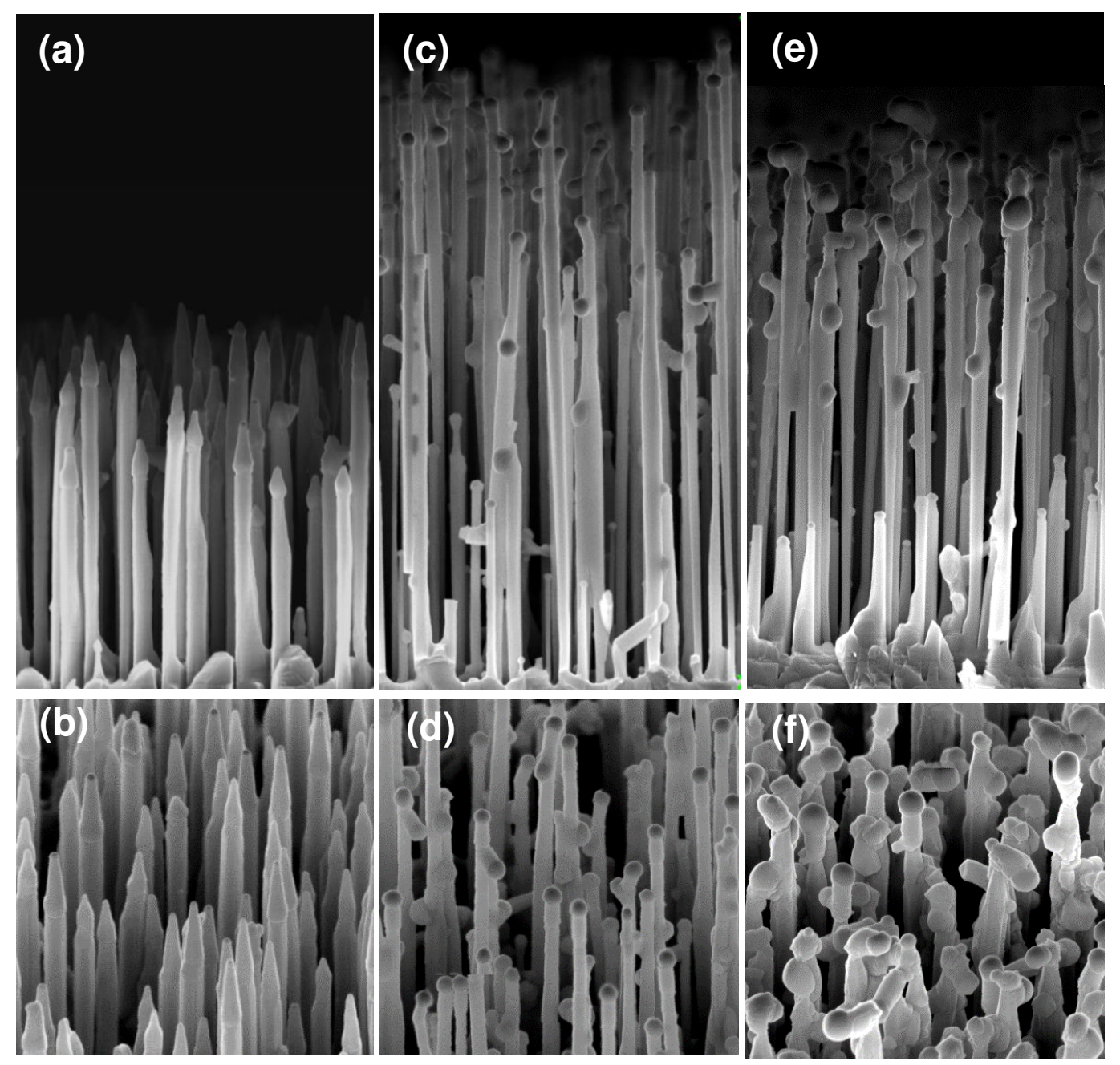

$500 \mathrm{~nm}$

Figure 1. SEM images of GaAs-Ga(As,Bi) core-shell nanowires grown on GaAs(111)B substrate. (a), (b) - sample 1 (1\% Bi); (c), (d) - sample 2 (4\% Bi); (e), (f) - sample 3 (4\% Bi and additional $(\mathrm{Ga}, \mathrm{Al}) \mathrm{As}$ shell in-between GaAs NW trunk and $\mathrm{Ga}(\mathrm{As}, \mathrm{Bi})$ shell. Upper panels cross-sectional views, lower panels $-45^{\circ}$ tilted views. The $500 \mathrm{~nm}$ scale bar plotted in the bottom part of the figure is common for all the panels.

A deeper insight into the NWs structure has been obtained by transmission electron microscopy (TEM) investigations. Morphology and structure of the NWs have been investigated using a FEI Titan 80-300 transmission electron microscope (TEM) operating at $300 \mathrm{kV}$ with a spherical 
aberration corrector of objective lenses in the HRTEM mode and a doubly corrected ARM200F microscope, operating at $200 \mathrm{kV}$. The elemental composition determination was carried out using EDAX $30 \mathrm{~mm} 2 \mathrm{Si}(\mathrm{Li})$ detector with a collection angle of 0.13 srad and a $100 \mathrm{~mm}^{2}$ Oxford Instruments windowless EDX detector installed within the Jeol ARM200F microscope.

For TEM investigation the NWs have been transferred mechanically onto copper grids covered with holey carbon films enabling imaging the entire NWs. The cross sections of the epoxy resin or platinum-carbon composite embedded NWs have been cut using a focused ion beam in Helios Nanolab 600 FIB as it is described in Ref. ${ }^{23}$.

\section{Results and Discussion}

Figure 2 shows TEM and scanning transmission electron microscopy (STEM) images of an individual $\mathrm{NW}$ from sample 1, grown with low Bi flux. Interestingly, pure ZB structure was revealed at the conical NW tip (Figure $2 \mathrm{~b}$ and $2 \mathrm{e}$ ) no distinct lines revealing SF or TB (twin boundaries) are visible. We infer that these $\mathrm{ZB}$ tips emerge due to the residual axial growth during $\mathrm{Ga}(\mathrm{As}, \mathrm{Bi})$ shell deposition. The pure $\mathrm{ZB}$ phase at the upper NW section is consistent with the reported dependence of GaAs NW phase on the NW tip-droplet contact angle ${ }^{24}$. The authors of Ref..$^{24}$ observed three different regions of droplet - NW tip contact angle ranges (here defined as $\beta$ ) inducing $\mathrm{WZ}$ or $\mathrm{ZB}$ structure during the $\mathrm{NW}$ growth. For low $\beta$ values $(80-100$ deg) the ZB phase is promoted, intermediate $\beta$ values $(100-125 \mathrm{deg})$ promote WZ phase, and higher $\beta$ values ( $125-140 \mathrm{deg})$ induce again the occurrence of ZB phase in the NW. As can be observed in Figure $2 \mathrm{~b}$, the droplet contact angles for the NW tip are in the low $\beta$ range, $90^{\circ}$ on the left and $100^{\circ}$ on the right side of the NW-droplet interface ( $\beta_{1}$ and $\beta_{2}$, respectively), which according to Ref. ${ }^{24}$ should promote ZB phase of GaAs NWs. 

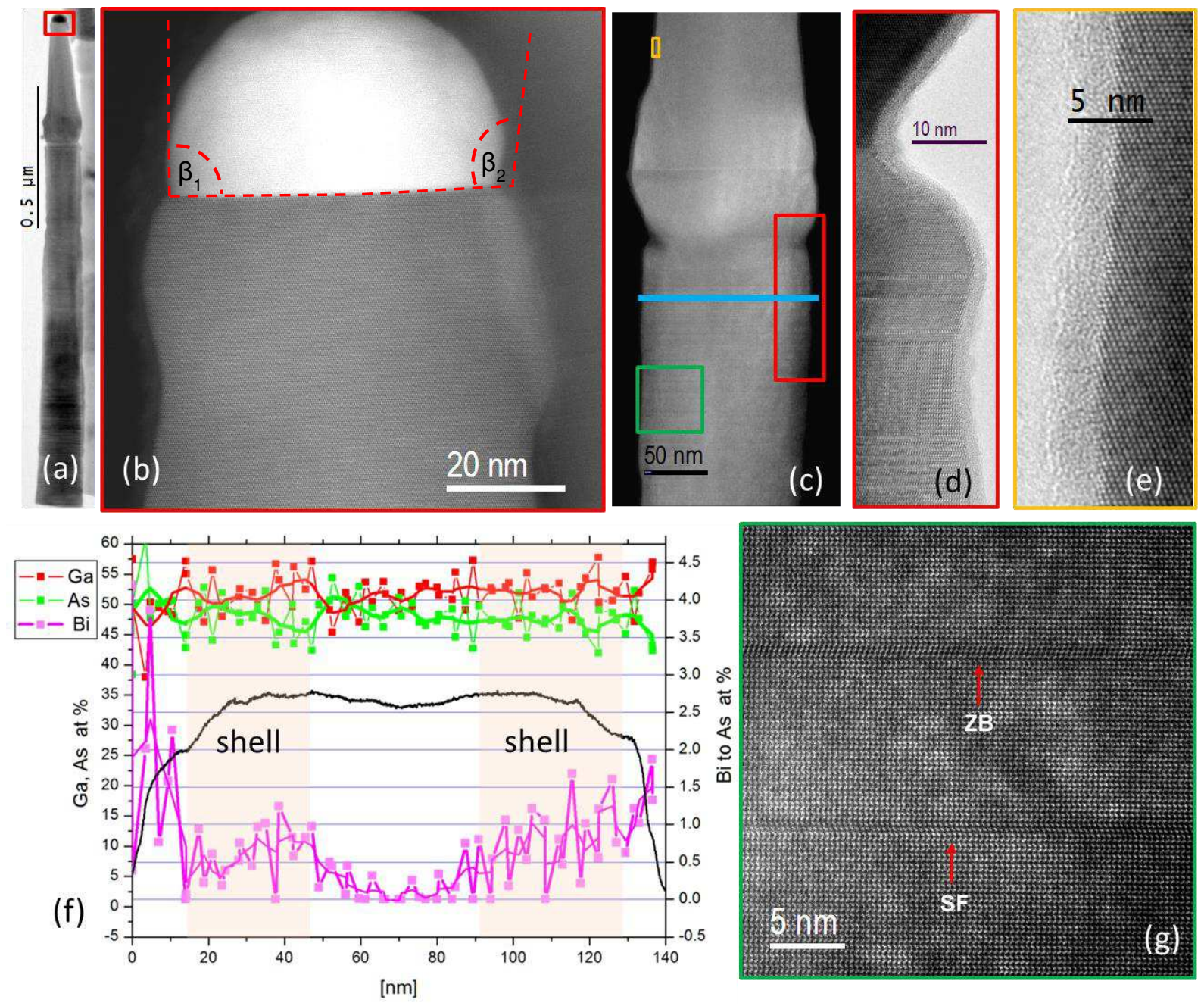

Figure 2. (a) TEM image of a $\mathrm{GaAs}-\mathrm{GaAs}_{0.99} \mathrm{Bi}_{0.01}$ core-shell nanowire (sample 1). Conical tip results from the residual axial growth during $\mathrm{Ga}(\mathrm{As}, \mathrm{Bi}$ ) shell deposition. (b) ADF-STEM image of the upper zinc-blende stacking fault free part of NW below gold catalyzer droplet. (c) STEM image of the transition part of the NW between GaAs core and the section which grows during $\mathrm{Ga}(\mathrm{As}, \mathrm{Bi})$ shell deposition. The conical tip is purely $\mathrm{ZB}$ and is free from stacking fault defects.

(d) HRTEM image showing progressive transition from WZ to pure ZB structure of the top NW part displayed in (e); (f) typical structure of the bottom part of the NW bellow the transition zone evidencing mainly WZ structure, the single SF and the 3 ML thick ZB inclusion are also visible. 
(g) EDS elemental distribution profile for As, $\mathrm{Bi}$ and Ga along the blue line together with the STEM intensity profile showing shape characteristic for the core -shell NW (due to the higher Z of $\mathrm{Bi}$ the shell is brighter).

The Bi content values exceeding 1at.\% at the surface of NWs (see the STEM intensity profile), are the quantification artefacts. Quantification is based on the Bi M-line whose intensity in the spectrum is clearly above the noise level. Also the As concentration drops to $99 \%$ with corresponding 1\% Bi content detected. Quantification based on Bi L-line gives higher values of about 2 at.\% but this line coincides with the As-K peak energy which can increase the apparent bismuth content due to not perfect deconvolution. The elemental distribution shown in Fig. $2 \mathrm{f}$ is expressed in at.\%, for $\mathrm{Ga}$ and $\mathrm{As}$, whereas the $\mathrm{Bi}$ concentration is normalized to the As atoms. From the Bi distribution and STEM-HAADF profile we can estimate the NW core diameter of $40 \mathrm{~nm}$ and the $\mathrm{Ga}(\mathrm{As}, \mathrm{Bi})$ shell thickness of $\sim 30-40 \mathrm{~nm}$. The maximum concentration of $\mathrm{Bi}$ is found at the NW side-wall surface (the amorphous "skin" of the NW - see Fig. S5 in the supplementary material) but the maximum concentration of $\mathrm{Bi} / \mathrm{As}$ in the shell is at the level of $\sim 1 \%$. Interestingly in the planar $\mathrm{ZB} \mathrm{Ga}(\mathrm{As}, \mathrm{Bi})$ layer grown together with sample 1 the $\mathrm{Bi}$ content evaluated from the $\mathrm{Ga}(\mathrm{As}, \mathrm{Bi})$ lattice parameter (see Fig. S1 in the supplementary material) amounts to $4.6 \%$, which proves much effective incorporation of Bi into planar ZB GaAs(100) than to WZ GaAs(11-20) NW sidewall planes.

In order to increase the $\mathrm{Bi}$ content in $\mathrm{Ga}(\mathrm{As}, \mathrm{Bi}) \mathrm{NW}$ shells, sample 2 was grown with much higher Bi flux intensity, than that used for sample 1. SEM images of sample 2 (figures 1c and 1d) show longer NWs in comparison to sample 1 (figure 1a and 1b). GaAs cores in sample 2 were 
grown for longer time ( $3 \mathrm{~h} 15 \mathrm{~min}$ vs $1 \mathrm{~h}$ growth time relevant for sample 1 ), hence the NW lengths reach up to $4.5 \mu \mathrm{m}$. The $\mathrm{Ga}(\mathrm{As}, \mathrm{Bi})$ shells were grown with $\mathrm{T}_{\mathrm{Bi}}=580{ }^{\circ} \mathrm{C}$ which corresponds to $\sim 4$ times higher Bi flux (sample 1 was grown with $\mathrm{T}_{\mathrm{Bi}}=540{ }^{\circ} \mathrm{C}$ ). The detailed TEM analyses of the NW collected from sample 2 are shown in Fig. 3. The main difference between samples 2 and 1 is the occurrence of droplets at the NW sidewalls in the former - see Fig.3a-3f. Apparently, the Bi excess, which is not incorporated into $\mathrm{Ga}(\mathrm{As}, \mathrm{Bi})$ shells, accumulates as droplets at the NW sidewalls. Additionally, a thin amorphous Bi layer can be observed at the NW sidewalls (Fig.S5 in the supplementary material). Moreover, as shown in Fig 3d, a substantial Bi content is also revealed in the catalytic droplet at the NW top (solidified into nanocrystal at room temperature TEM investigations). The top droplet clearly consists of the two distinct parts. The part appearing as brighter seems to be embedded in the bigger, darker one (see Fig. 3d). The EDS analysis shows that the larger droplet consists of pure, solidified Bi. The elemental composition of the smaller part composition suggests that it contains a metastable $\mathrm{Au}_{\sim 0.40} \mathrm{Bi}_{\sim 0.60}$ phase $^{6}$. The side droplet shown in Fig, $3 \mathrm{a}$ and $3 \mathrm{~b}$ contains mainly Bi with a small admixture of As (up to 3 at.\%). This droplet crystallized just below the transition region from mainly WZ to the pure ZB part. The details of this transition zone are shown in Fig. 3b and 3c. This WZ-ZB transition is quite abrupt, along about $5 \mathrm{~nm}$ long NW section. The upper ZB part is free form SF or TB defects. Figure 3d shows another NW also containing top and side droplet. Similarly to the first NW shown in Fig 3a and 3b, the top droplet also consists of two parts: pure $\mathrm{Bi}$ and $\mathrm{Au}$-rich $\mathrm{Au}_{2} \mathrm{Bi}$ phase. Also in this case the pure $\mathrm{ZB}$ structure part is about $100 \mathrm{~nm}$ long. The details of this part are shown in Fig. S4 of the supplementary material, revealing that it hosts an oblique $\mathrm{SF}$ which starts at the WZ-ZB transition zone and ends at $\mathrm{Au}_{2} \mathrm{Bi} / \mathrm{Bi}$ interface. This suggests that $\mathrm{Au}_{2} \mathrm{Bi}$ phase nucleated at the $\mathrm{SF}$ and emerged during residual axial growth of this 
upper (ZB) NW part. Hence this part grew in a dual VLS and (vapor-solid-solid) VSS mechanism.

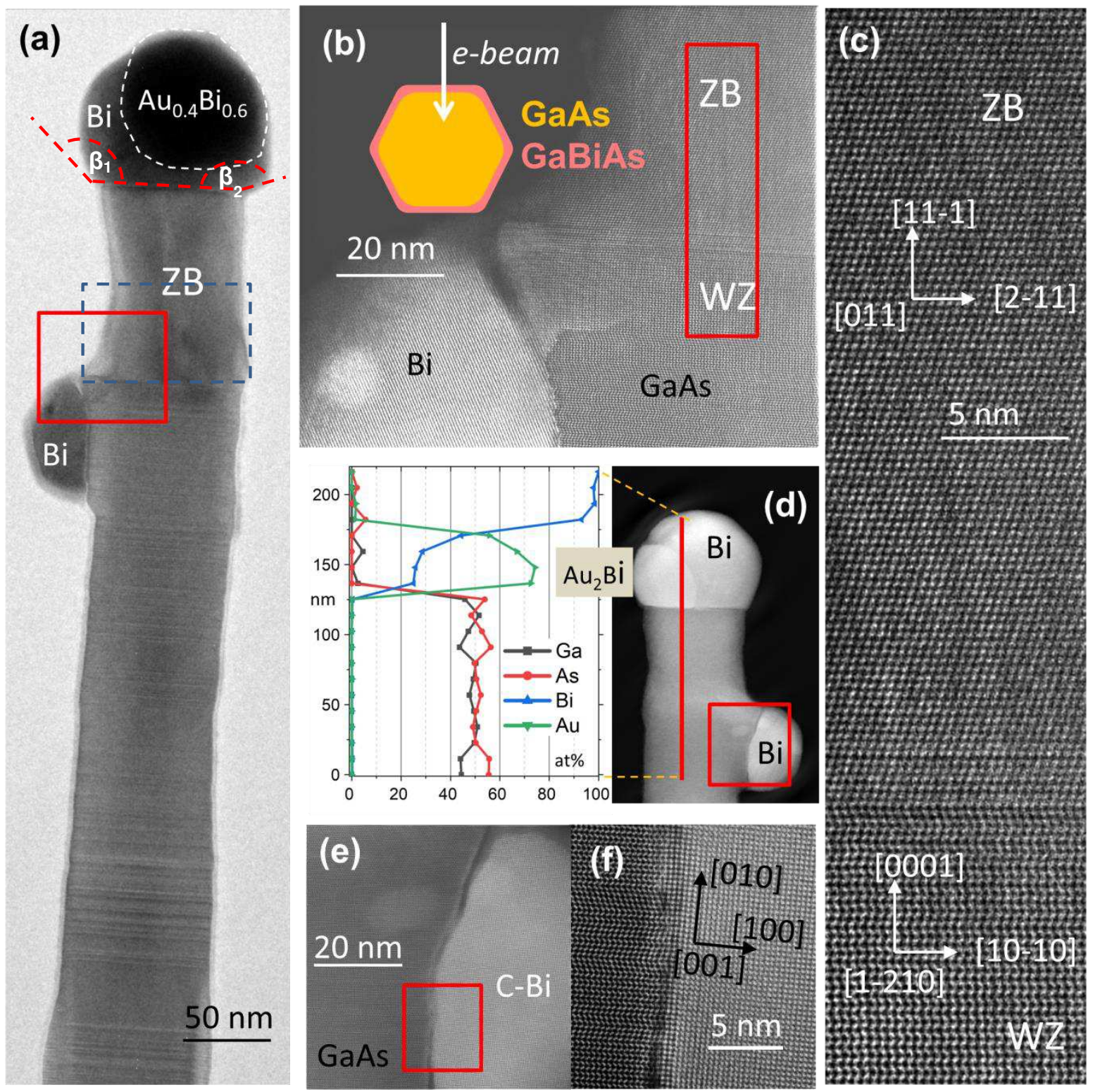

Figure 3. (a) HRTEM of sample 2; (b) STEM of the frame in (a) a diagram shows orientation of the facets in relation to the e-beam; (c) STEM of the frame in b; (d) STEM image of another NW and EDS elemental concentration profile along the red line; (e) STEM zoomed part of the frame from d; (f) zoomed part of the frame in (e). 
The side Bi droplet crystallized in the uncommon cubic Bi phase ${ }^{25}$ with the lattice parameter of $3.17 \AA$ (see Fig. 3f). The (010) planes in the Bi droplet are in the epitaxial relation to WZ GaAs planes $\mathrm{d}_{(0002)}$. The Bi lattice planes in vertical direction inside the droplet visible in STEM images (see Fig. 3f) have the interplanar distance $\mathrm{d}_{(010)}=3.285 \mathrm{~A}$ but in the radial direction the spacing $d_{(100)}$ equals to $3.33 \mathrm{~A}$. These mean that the measured lattice parameter is about $3 \%$ bigger then that reported in Ref. $^{25}$ and we also detect a tetragonal distortion of $1.7 \%$. The lattice planes of $\mathrm{Bi}$ in the (solidified) side droplets are rotated clockwise by 1 deg. in relation to the core WZ GaAs lattice.

In contrast to sample 1 with pure top Au droplets with diameters of $\sim 55 \mathrm{~nm}$ and a $400 \mathrm{~nm}$ long conical ZB parts below the top droplet of sample 2, (see Fig. 3a) is much larger - about 100 $\mathrm{nm}$ in diameter, but the length of the top ZB segment is much shorter $(\sim 110 \mathrm{~nm}$.). From now on, we will assign the name "NW neck" to the top ZB segment of the NW which results from the residual axial growth during the $\mathrm{Ga}(\mathrm{As}, \mathrm{Bi})$ shell deposition (as discussed above). The shape differences of the NW necks in samples 1 and 2 can be explained as follows. In both cases, two growth modes of the NW neck occur simultaneously: (i) axial growth and (ii) radial growth. In sample 1, the catalytic droplet is small, without any $\mathrm{Bi}$, since the $\mathrm{Bi}$ flux is low and all impinging Bi is incorporated into the NW shells (see Fig.1a,b and Fig.2a). It is well-known, that when the growth is controlled by the diffusion flux of adatoms along the NW sidewalls towards the top, the axial NW growth rate is inversely proportional to the size of the catalytic droplet ${ }^{26}$. Smaller droplets in sample 1 induce faster residual axial growth during the $\mathrm{Ga}(\mathrm{As}, \mathrm{Bi})$ shell deposition. The shell growth time in sample 1 was 1 hour, resulting in $400 \mathrm{~nm}$ long conical ZB NW neck. The Ga flux applied during the shell growth corresponded to the high temperature axial growth rate of the GaAs NW trunk equal to $1 \mu \mathrm{m} / \mathrm{h}$. The axial growth rate of the NW neck during the 1 
hour shell deposition is 2.5 times slower due to the low temperature of the shell growth (about $300{ }^{\circ} \mathrm{C}$ ), and competing mechanism of the radial growth. The conical shapes are typical for NWs grown at low temperatures, where the axial and radial growth modes occur simultaneously ${ }^{16}$. Here, the top droplet is $\sim 100 \mathrm{~nm}$ in diameter; twice the size of the droplet in sample 1, but the ZB NW neck is only $\sim 120 \mathrm{~nm}$ long (in contrast to $400 \mathrm{~nm}$ long neck of sample 1). The shell deposition time for sample 2 was $0.5 \mathrm{~h}$ (1h for sample 1), with the same Ga flux intensity, corresponding to a planar growth rate of $0.2 \mu \mathrm{m} / \mathrm{h}$. The different neck shape in Sample 2 (in comparison to Sample 1) is most probably correlated to the larger droplet size upon exposure to high $\mathrm{Bi}$ flux during the $\mathrm{Ga}(\mathrm{As}, \mathrm{Bi})$ shell growth, with the droplet gradually accumulating more $\mathrm{Bi}$, which compensates the tapered shape (even reversed tapering can be inferred from Fig.3a induced by the low growth temperature). Standard NW tapering was observed in the first $50 \mathrm{~nm}$ of the NW neck (see section enclosed the blue dashed rectangle in Figure 3a, whereas a slightly inverse tapering is visible in the section above. The common tapering effect associated with the radial growth, most pronounced at the lower part of the NW neck was compensated by the increasing diameter of the upper neck part, due to increasing top droplet size and Bi content. This effect can be observed in the two different NWs shown in Fig. 3a and 3d. The measured droplet contact angles $\beta_{1}$ and $\beta_{2}$ are equal to 135 and 200 deg. respectively in the top NW drop section shown in Fig. 3a. According to the in-situ TEM NW growth investigations shown in Ref. [24], the contact angles higher than about $125 \mathrm{deg}$. promote the ZB GaAs NW phase.

To get better insight into the formation and morphology of $\mathrm{Ga}(\mathrm{As}, \mathrm{Bi})$ shells, in the case of sample 3, a 15 nm thick (Ga,Al)As shells, with 30\% Al, had been grown on the GaAs NW core before the $\mathrm{Ga}(\mathrm{As}, \mathrm{Bi})$ shell growth. The $(\mathrm{Ga}, \mathrm{Al}) \mathrm{As}$ shells were deposited at $400{ }^{\circ} \mathrm{C}$. The lighter (Ga,Al)As layer contrast in ADF-STEM images (see Fig. 4b) allows to unequivocally identify 
the $\mathrm{Ga}(\mathrm{As}, \mathrm{Bi})$ shells both in the plan-view and cross sections images of the NW. EDS compositional distribution across the WZ area of the NW shown in Fig. $2 \mathrm{f}$ reveals that the NW shell contains about $1 \% \mathrm{Bi}$
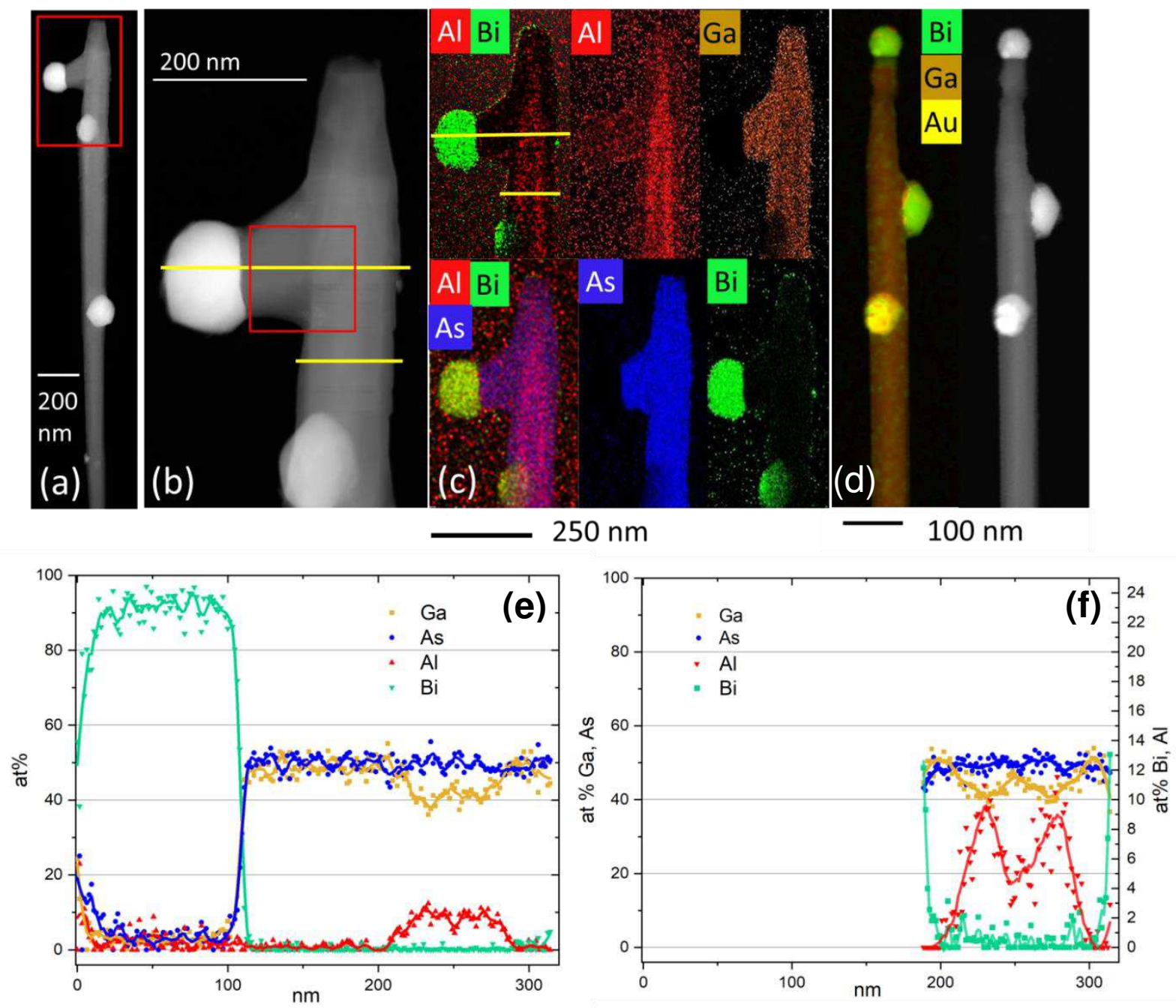

Figure 4. (a), (b) - TEM images of the upper GaAs-(Ga,Al)As-Ga(As,Bi) core-double shell NW part with Bi-catalyzed side branch (the very top part with the top catalyzing droplet has been broken during the NW collection), the side Bi droplet catalyzes the branch growth; (c) EDS composition maps visualizing distribution of the elements: $\mathrm{Ga}, \mathrm{Al}, \mathrm{As}, \mathrm{Bi}$ in the NW section shown in (b); (d) - EDS composition map of another NW picked from Sample 3, showing Au 
droplet, which slid downwards and was replaced by the Bi droplet at the NW top; (e), (f) - EDS line scans showing distribution of elements along yellow lines marked in (b) and (c).

The Ga(As,Bi) shells were grown in the same conditions (growth temperature, Bi and Ga fluxes, As/Ga flux ratio) as sample 2, but for slightly longer time (45 min for sample 3, versus $30 \mathrm{~min}$ for sample 2). The $50 \%$ longer $\mathrm{Ga}(\mathrm{As}, \mathrm{Bi})$ growth under $\mathrm{Bi}$ excess conditions leads to a more pronounced Bi segregation forming more droplets at the NW sidewalls. Figure 4 summarizes the compositional analysis carried out for two representative NWs from sample 3. Two different upper GaAs-(Ga,Al)As-Ga(As,Bi) core-double shell NWs are displayed. The first one shown in Fig 4 a,b,c has missing top. The HRSTEM images of the Bi droplet catalyzed side branch and branch-trunk regions are shown in Fig.S6 of the Supporting information. Similarly to the case of sample 2 the residual axial growth during crystallization of $\mathrm{Ga}(\mathrm{As}, \mathrm{Bi})$ shell produces distinct necks (visible also in Fig 1e, and 1f). This neck part can easily be broken during mechanical transfer of NWs to the TEM grid. A complete NW is shown in Fig. $4 \mathrm{~d}$ and Fig. S7 in the supplementary material. The EDS maps (Fig. 4c) and profiles (Fig. 4e,f) reveal the inner GaAs core and (Ga,Al)As shell. This data let us conclude that during the growth of (Ga,Al)As shell also axial (Ga,Al)As growth was continued over the length of $\sim 200 \mathrm{~nm}$. After the temperature drop necessary to grow $\mathrm{Ga}(\mathrm{As}, \mathrm{Bi})$ shell the gold droplet was replaced by the $\mathrm{Bi}$ one, and axial growth continued in both axial and slightly off-axis (random) directions; as can be seen in Fig.1e,f. 
The accumulation of liquid $\mathrm{Bi}$ at the sidewall surface resulted in formation of additional Bi-rich droplets which start to catalyze the secondary branches reproducing the trunk structure twinning $\mathrm{ZB}$ parts and $\mathrm{SF}$ in $\mathrm{WZ}$ parts of the core, as shown in Fig.S6 in the Supporting Information. Replacing the Au droplet by the Bi one at the NW top can be explained as follows. The temperature decrease down to $300{ }^{\circ} \mathrm{C}$ for $\mathrm{Ga}(\mathrm{As}, \mathrm{Bi})$ shell deposition caused crystallization of $\mathrm{Au}$ at the NW top, but after delivery of $\mathrm{Bi}$ in large amount, the external part of hitherto crystalized $\mathrm{Au}$ droplet became liquid. In the $\mathrm{Au}-\mathrm{Bi}$ system, there are two eutectic points $371{ }^{\circ} \mathrm{C}$ and $241{ }^{\circ} \mathrm{C}$, depending on the Bi concentration ${ }^{6}$. We infer that the $241{ }^{\circ} \mathrm{C} \mathrm{BiAu}$ eutectic was formed and the surface of the gold nanocrystal at the NW top became liquid, which allowed the droplet to move and float down as shown in the EDS map in Fig. 4d. High magnification ADFSTEM image of the Bi-droplet catalyzed branch, corresponding to the region marked by the red square in Fig.4b, is shown in Fig.S6 in the Supporting Information.

Figure 5 shows a STEM image and EDS elemental composition maps taken from a $75 \mathrm{~nm}$ thick FIB cross-section of the GaAs-(Ga,Al)As-Ga(As,Bi) NW in sample 3. High magnification ADF-STEM images were obtained using a HAADF detector with angular collection between $\alpha_{\min }=65 \mathrm{mrad}$ and $\alpha_{\max }=210 \mathrm{mrad}$. The thickness of the analyzed NW cross-section is measured using position averaged convergent beam electron diffraction (PACBED) technique as described in the Supporting information. Under these recording conditions, the HAADF images are sensitive to the average atomic number $(\mathrm{Z})$ of the column (see Fig 5a). The hexagonal shape of the GaAs core is reproduced in the successive shells. However, the last LT-GaAs shell does not develop sharp corners. The measured thicknesses of the $(\mathrm{Ga}, \mathrm{Al}) \mathrm{As}$ shells on all six NW sidewalls are similar, equal to $18-20 \mathrm{~nm}$. The local contrast maxima correspond to projections of $\mathrm{A}, \mathrm{B}, \mathrm{C} \mathrm{ZB}$ stacking position of the $(\mathrm{Ga}, \mathrm{Al}) /(\mathrm{As}, \mathrm{Bi})$ columns. In this particular image all the 
contrast maxima have similar intensity what could suggest that this cross-section contains the ZB segment. However, we cannot exclude that this segment consists partially of the WZ structure as well. One can notice that WZ structure, described with ...ABAB... stacking along 0001 crystallographic direction, changes its sequence once experiencing intrinsic stacking fault (ISF). Introducing one or two cubic planes (known as ISF1 or ISF2) results in ...ABABCBCBC... or ...ABABCACACA... stacking, respectively. This seemingly small deviation from the pure wurtzite structure has its consequence visible on the (0001) projection, i.e. it cannot be distinguished from $\mathrm{ZB}$ structure since all ABC planes are present.
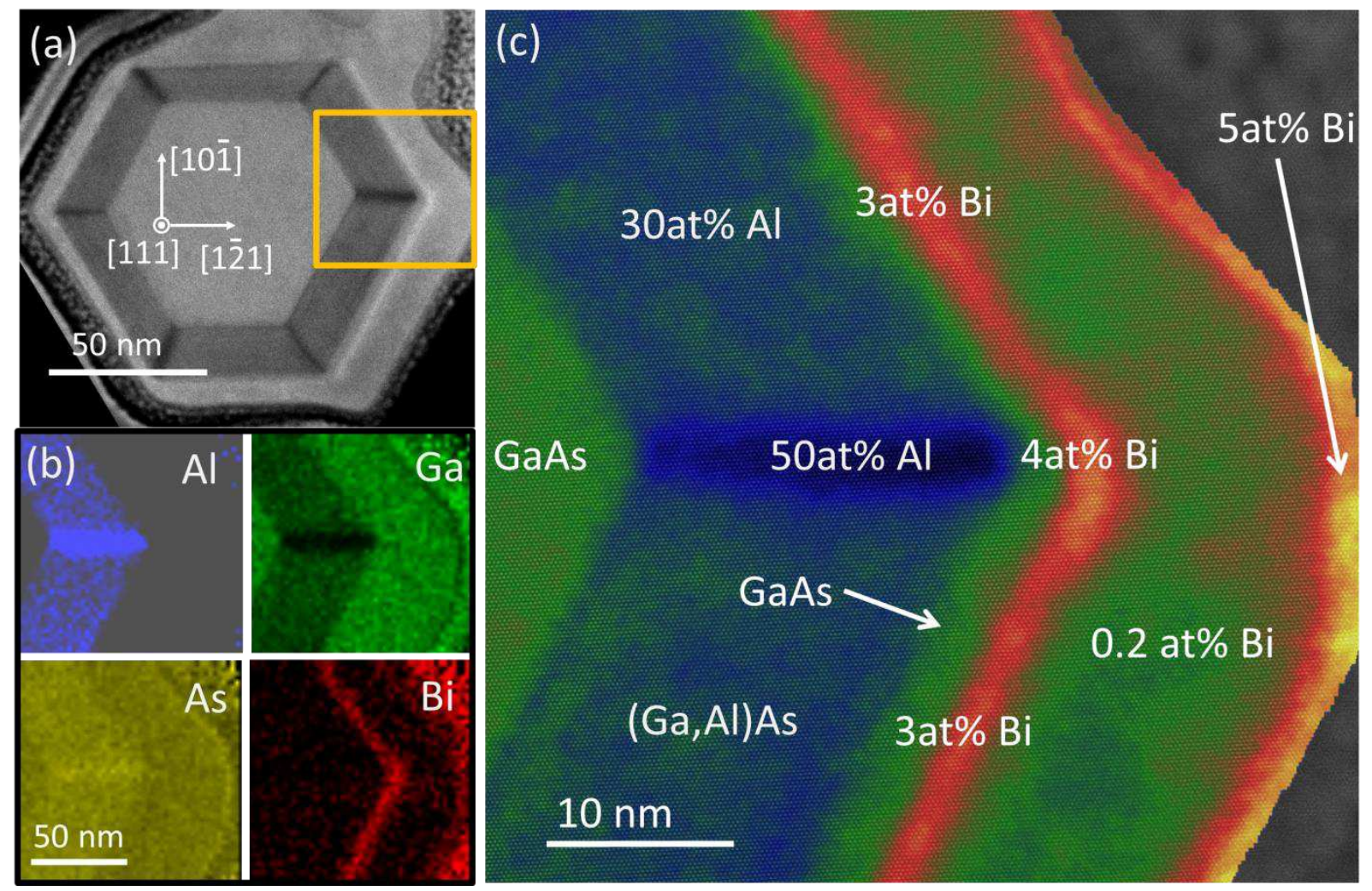

Figure 5. Analysis of a thin cross-section of ZB GaAs-(Ga,Al)As-Ga(As,Bi) NW segment. (a) ADF-STEM images with frame indicating position of EDS map; (b) map of Al, Ga, As, Bi elements distribution maps; (c) semi-quantitative EDS analysis of the edge of the ZB NW; (d) color coded STEM image according to EDS signal and STEM intensity. 
A characteristic dark contrast features lying along the $<112>$ crystallographic direction have been observed as shown in Fig 5a. These dark lines running from the GaAs core corners have already been reported ${ }^{27,28,29}$ for NWs with $\mathrm{ZB}(\mathrm{Ga}, \mathrm{Al}) \mathrm{As}$ shells where high $\mathrm{Al}$ concentration, i.e. $\sim 50 \%$ was detected in these regions and 30\% $\mathrm{Al}$ elsewhere. Nanowires with $\mathrm{WZ}$ (Ga,Al)As shells reported e.g. by Leandro et. al. ${ }^{30}$ have not been studied in this aspect so far, to our knowledge. Outside the (Ga,Al)As shell, the $4 \mathrm{~nm}$ thick $\mathrm{Ga}(\mathrm{As}, \mathrm{Bi})$ layer with higher $\mathrm{Bi}$ concentration appears as a brighter region in Fig 5a (red in Fig 5c). Moreover, a second thin, bright layer is visible at the very edge of the NW (orange in Fig. 5c). It should be noted that the $\mathrm{Ga}(\mathrm{As}, \mathrm{Bi})$ shell thickness is homogenous for most of the NW sidewalls, except the ones being in the closest neighborhood of other NWs, known as MBE shadowing effect. Figure 5b shows the EDS elemental composition analysis. This analysis was performed for the ZB NW like the one, shown in panel (a) of this figure, enabling a direct correlation between STEM image intensity and the concentration of elements visualized in the EDS maps. The Al and Ga maps clearly show the enhancement of $\mathrm{Al}$ concentration in the darker areas of the STEM images, with $(\mathrm{Ga}, \mathrm{Al}) \mathrm{As}$ radial segregation running along $\langle 112>$ direction. Bi distribution maps corroborate the presence of continuous Bi-rich thin inner shell and Bi-enriched NW external surfaces. Figure 5c shows a false-colored HR-STEM image obtained by combining information from EDS maps and intensities of STEM images. The STEM intensity was scaled based on the EDS results in the following way: in the case of $\mathrm{Al}$ and $\mathrm{Ga}$ the average ratio of $\mathrm{Al} / \mathrm{Ga}$ from the shell region and from high $\mathrm{Al}$ content area were referred to the average STEM intensity in the same area. Then, the linear dependence of $\mathrm{Al} / \mathrm{Ga}$ and STEM intensity was assumed. In the case of $\mathrm{Bi}$, a similar procedure was applied but the average values for the middle of the Bi rich area were taken into 
account. For calculating the average chemical composition in the given area, the sum of spectra was used for elements quantification. Finally, the elemental composition maps were plotted with higher spatial resolution than original EDS maps of 50x50 pixels. The average Al concentration in the $(\mathrm{Ga}, \mathrm{Al}) \mathrm{As}$ shell is at the level of 30 at.\% with respect to $\mathrm{Ga}$. The maximum concentration of 50 at.\% $\mathrm{Al}$ is reached at the corners of the $(\mathrm{Ga}, \mathrm{Al}) \mathrm{As}$ shell. The average concentration of $\mathrm{Bi}$ with respect to As reaches 3 at.\% but it is higher (4 at.\%) at the corners of the hexagon. The highest $\mathrm{Bi}$ concentration (5 at.\%) is measured at the NW sidewall surface, however some $\mathrm{Bi}$ atoms are not embedded into GaAs lattice but remain at the surface in the form of amorphous layer. The average concentration of $\mathrm{Bi}$ between the $\mathrm{Bi}$ rich layer close to the inner $(\mathrm{Ga}, \mathrm{Al}) \mathrm{As}$ shells and the sidewall surface drops to small values generally less then 1at \% (green areas in Fig $5 \mathrm{c}$ ). However the Bi content in the areas with Bi concentration smaller than $\sim 0.2$ at $\%$ which is in our case under the detection limit (blue areas in green) is only roughly estimated, however the intensity of Bi-M line in the average spectrum is still above the noise level. Additionally, between the (Ga,Al)As shell and Bi-rich shell, the pure GaAs region with a thickness $\sim 1-2 \mathrm{~nm}$ is detected. Such a thin GaAs shell was intentionally deposited to avoid (Ga,Al)As surface contamination during the relatively long growth break required to stabilize a low temperature of $\mathrm{Ga}(\mathrm{As}, \mathrm{Bi})$ shell deposition $\left(300{ }^{\circ} \mathrm{C}\right)$ as well as to decrease and stabilize $\mathrm{As}_{2}$ flux to close-to stoichiometric value to the $\mathrm{Ga}$ one. On other hand, the presence of such a layer clearly indicates that Bi bulk diffusion does not occur during the LT growth of the $\mathrm{Ga}(\mathrm{As}, \mathrm{Bi})$ shell. The perimetric fluctuations of Bi concentration in the shell in the 2-5 nm scale are visible in Fig. 5c. However, averaging the information over $70-80 \mathrm{~nm}$ thick specimen blurs the localized $\mathrm{Bi}$ concentration fluctuations or clustering. 

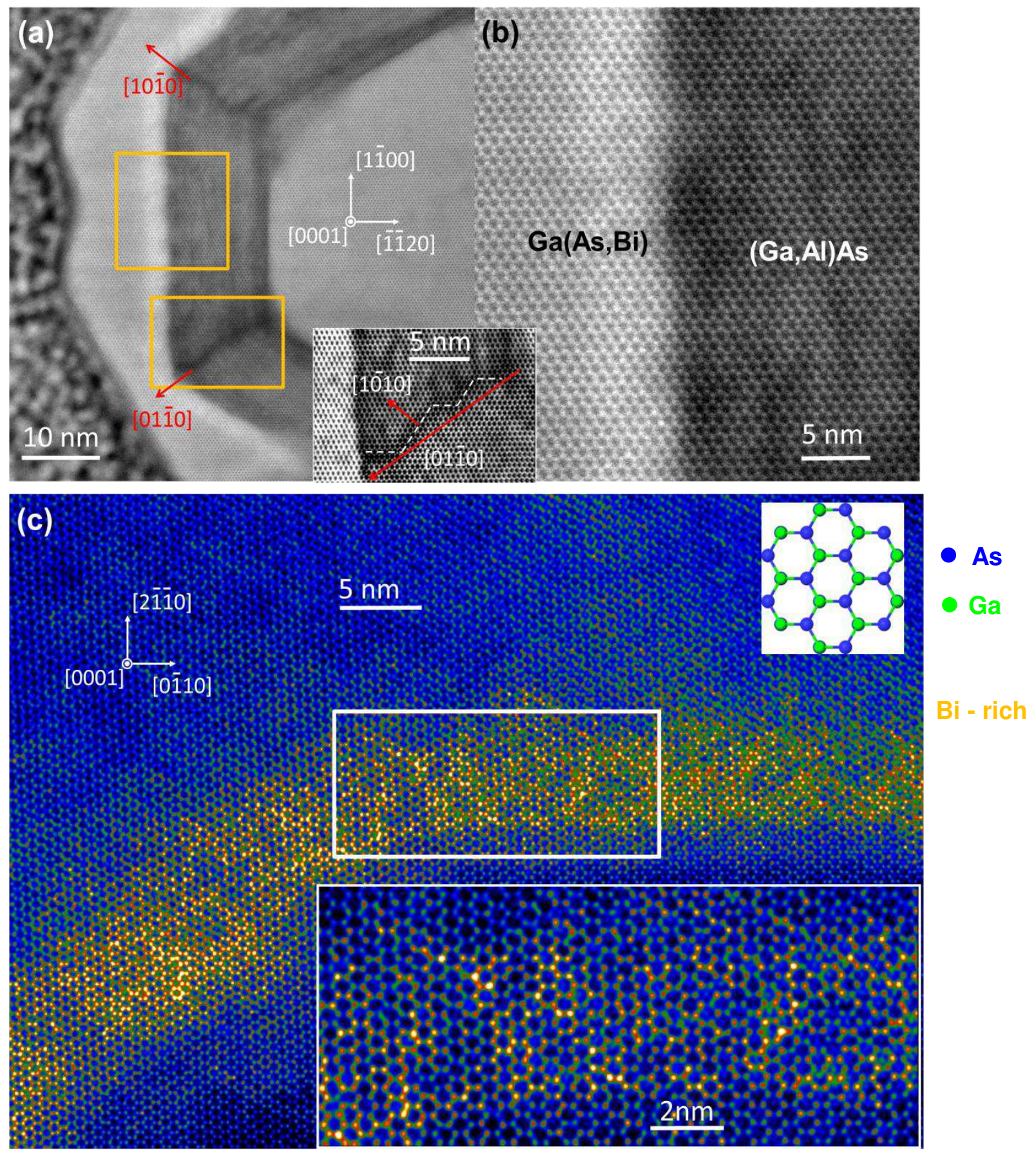

Figure 6. The analysis of a thin cross-section of the WZ NW segment. (a) - HR-STEM image acquired with the camera length of $73 \mathrm{~mm}$; (b) - zoomed part of the interface between (Ga,Al)As and $\mathrm{Ga}(\mathrm{As}, \mathrm{Bi})$ shells; the inset to (a) and (b) - zoomed filtered image showing details of $\mathrm{Al}$ segregation at the NW corner; (c) HR-STEM (in false colors) of about $20 \mathrm{~nm}$ thick cross-section 
of Bi-rich shell; yellow color represents Bi-rich atomic columns (averaged over 20-nm thick specimen)

Figure 6 shows a cross-sectional TEM image along the [0001] direction recorded in the WZ section of a NW from sample 3. In this case, the WZ phase can be easily identified due to fact that the only contrast maxima corresponding to ...ABAB... stacking are visible. In this case, the hexagonal shape of the core is also preserved. The 5-6 nm thick Bi-rich shell can be distinguished. This shell appears homogenous for all three NW facets visible in the image. We estimate that the specimen thickness is about $90 \mathrm{~nm}$, so the fine fluctuations cannot be detected. However, the segregation of $\mathrm{Al}$ is clearly visible inside the (Ga,Al)As shell. We also detect $\mathrm{Al}$ segregation at the edges of the hexagon, never reported before for WZ NWs. However, the segregation shown in the inset to Fig.6a,b looks differently than that occurring in ZB (Ga,Al)As NW sections, reported earlier ${ }^{27}$. In our case, the high Al concentration regions have zigzag shapes $^{27}$. Slightly similar zig-zag corner-line shapes were observed e.g. for P-rich regions of ZB $\mathrm{Ga}(\mathrm{As}, \mathrm{P}) / \mathrm{GaAs}$ coaxial $\mathrm{NWs}^{31}$. The structural details of this Al-enhanced region are shown in the filtered image (inset to Fig. 6) where the zigzag shape, Al-rich path (dashed line) does not follow solely radial [01-10] direction but consists of two crystallographic directions [10-10] and [1-100]. This difference, with respect to the (Ga,Al)As NW shell of ZB structure is related to the lower symmetry of WZ structure, where the diffusion paths can be limited in comparison to the ZB one. The WZ NW cross-section shown in Fig. 6 is the thinnest specimen prepared with the FIB technique without its significant amorphization. We estimate the thickness of this crosssection to be less than $20 \mathrm{~nm}$. Hence the local fluctuations of Bi atoms start to be visible with STEM and are much higher than those in the Bi-poor LT-GaAs shell. The Bi-rich areas are 
generally random but some texture in the radial direction can be distinguished; areas with the diameters of 1-2 nm can also be seen. In the cross-sections of both WZ and ZB parts of a NW collected from sample 3, we notice inhomogeneous concentration of $\mathrm{Bi}$ in the radial direction. Similar Bi concentration profile was also observed in the MBE-grown planar $\mathrm{Ga}(\mathrm{As}, \mathrm{Bi})$ layers, with $\mathrm{Bi}$ concentration exponentially dropping along the growth direction at first $25 \mathrm{~nm}$ $\mathrm{Ga}(\mathrm{As}, \mathrm{Bi})$ film thickness and then stabilizing at a constant level ${ }^{32}$. In the core-shell NW heterostructures investigated here the thickness of $\mathrm{Ga}(\mathrm{As}, \mathrm{Bi})$ shells is in the range of $14-26 \mathrm{~nm}$, hence the same effect can occur. Moreover, as revealed by the Monte Carlo simulations of Bi incorporation and droplets formation during the $\mathrm{MBE}$ growth of $\mathrm{Ga}(\mathrm{As}, \mathrm{Bi})$, once the surface $\mathrm{Bi}$ droplets are formed the incorporation of Bi into the growing film decreases considerabely ${ }^{15}$. In our case, both effects can occur. In the cross-section of a ZB NW part we also observe the enhanced Bi content at the outer NW region close to the sidewall surface and at the outer NW corner.

\section{Concusions}

In conclusion we have investigated $\mathrm{Bi}$ incorporation into predominantly wurtzite GaAs(Ga,Al)As-Ga(As,Bi) core-shell nanowires grown by molecular beam epitaxy. At low Bi content of $1 \%$ the NWs are smooth, with no additional features at their sidewalls, whereas at the attempted Bi content of 4\%, the droplets are formed at the NW sidewalls. At the NW tops Bi merges with the Au catalyst and induces (in the NWs cooled down to room temperature) the phase separation into $\mathrm{Au}_{2} \mathrm{Bi}$ and pure $\mathrm{Au}$, however with undisturbed spherical top nanoparticles shapes. In the cross section of the core multishell GaAs-(Ga,Al)As-Ga(As,Bi) NWs we have detected radially inhomogeneous distribution of $\mathrm{Bi}$. The enhanced $\mathrm{Bi}$ content occurs at the inner 
interfaces of $\mathrm{Ga}(\mathrm{As}, \mathrm{Bi})$ shells and on their outer surfaces (NW sidewalls). Additionally in the WZ (Ga,Al)As shells we have observed, so far unnoticed zigzag-shaped enhanced Al content regions along the lines extending radially out of the hexagonal WZ NW corners. In the NWs with mixed $\mathrm{ZB}-\mathrm{WZ}$ axial structure the preferential location of the side Bi droplets at the WZ NW sections was evidenced. Such Bi droplets induce the growth of WZ GaAs branches perpendicular to the NW trunks. The catalyzing action of Bi droplets, with preferential location at wurtzite nanowire segments, enables controlled formation of branches in the GaAs NWs with mixed WZZB structure, since the structural change between both GaAs polytypes can be induced by alterations of the NW growth conditions.

\section{Supporting Information:}

XRD spectrum, SEM and TEM images of a planar $\mathrm{Ga}(\mathrm{As}, \mathrm{Bi})$ layer grown together with GaAs-Ga(As,Bi) core shell nanowires (Sample 1); additional TEM images of NWs and NW cross-sections including EDS composition maps (Samples 2 and 3).

\section{References}

1. Pillai, M. R., Kim, S. S., Ho, S. T., Barnett, S. A. Growth of $\operatorname{In}_{x} \mathrm{Ga}_{1-\mathrm{x}} \mathrm{As} / \mathrm{GaAs}$ heterostructures using Bi as a surfactant. J. Vac. Sci. Technol. B 18, 1232-1236 (2000).

2. Usman, Muhammad, et al. Impact of alloy disorder on the band structure of compressively strained GaBi $\mathrm{As}_{1_{-\mathrm{x}}}$ Phys. Rev. B 87, 115104 (2013). 
3. Oe, Kunishige, \& Okamoto, Hiroshi. New semiconductor alloy $\mathrm{GaAs}_{1-\mathrm{x}} \mathrm{Bi}_{\mathrm{x}}$ grown by metal organic vapor phase epitaxy. Jpn. J. Appl. Phys. 37, L1283-L1285 (1998).

4. Tixier, S., Adamcyk, M., Tiedje, T., Francoeur, S., Mascarenhas, A., Wei, Peng., \& Schiettekatte, F. Molecular beam epitaxy growth of $\mathrm{GaAs}_{1-\mathrm{x}} \mathrm{Bi}_{\mathrm{x}}$ Appl. Phys. Lett. 82, 2245$2247(2003)$.

5. Wang, Shumin., \& Lu, Pengfei (Eds.) Bismuth-containing alloys and nanostructures. Springer Series in Materials Science, 285 (2019).

6. Okamoto, H. Supplemental literature review of binary phase diagrams: Bi-Ga, Bi-Y, Ca-H, Cd-Fe, Cd-Mn, Cr-La, Ge-Ru, H-Li, Mn-Sr, Ni-Sr, Sm-Sn, and Sr-Ti. J. Phase Equilibria Diffus. 36, 292-303 (2015).

7. Masnadi-Shirazi, M., Lewis, R. B., Bahrami-Yekta, V., Tiedje, T.; Chicoine, M., \& Servati P. Bandgap and optical absorption edge of $\mathrm{GaAs}_{1-\mathrm{x}} \mathrm{Bi}_{\mathrm{x}}$ alloys with $0<\mathrm{x}<17.8 \%$. J. Appl. Phys., 116, 223506 (2014).

8. Wang, Lijuan. et al. Novel dilute bismide, epitaxy, physical properties and device application. Crystals 7, 63 (2017).

9. Huang, Huaqing., Liu, Jianpeng., \& Duan, Wenhui. Nontrivial Z2 topology in bismuthbased III-V compounds. Phys. Rev. B 90, 195105 (2014).

10. Hsu, Chia-Hsiu, et al. Growth of a predicted two-dimensional topological insulator based on InBi-Si(111)- $\sqrt{7} \times \sqrt{ } 7$. Phys. Rev. B 98, 121404(R) (2018). 
11. Nicolaï, Laurent, et al. Topological surface states from ordered InBi crystals. arXiv:1806.03061 (2018).

12. Fang, Zhenyao., Gao, Heng., Venderbos, Jörn W. F., \& Rapp, Andrew M., Ideal near-Dirac triple-point semimetal in III-V semiconductor alloys. Phys. Rev. B 101, 125202 (2020).

13. Caroff, Philippe., Bolinsson, Jessica., and Johansson, Jonas. Crystal phases in III-V nanowires: from random toward engineered polytypism. IEEE J. Sel. Top. Quantum Electron. 17, 829-846 (2011).

14. Lewis, R. B., Masnadi-Shirazi, M., \& Tiedje, T. Growth of high Bi concentration $\mathrm{GaAs}_{1-\mathrm{x}} \mathrm{Bi}_{\mathrm{x}}$ by molecular beam epitaxy. Appl. Phys. Lett. 101, 082112 (2012).

15. Rodriguez, Garrett V. and. Millunchick, Joanna, M. Predictive modeling of low solubility semiconductor alloys. J. Appl. Phys. 120, 125310 (2016).

16. Harmand, J.C., Tchernycheva, M., Patriarche, G., Travers, L., Glas, F., Cirlin, G. GaAs nanowires formed by Au-assisted molecular beam epitaxy: Effect of growth temperature. $J$. Crystal Growth 301-302, 853-856 (2007).

17. Sadowski, J. et al. GaAs:Mn nanowires grown by molecular beam epitaxy of (Ga,Mn)As at MnAs segregation conditions. Nano Lett. 7, 2724-2728 (2007).

18. Rudolph, A. et al. Ferromagnetic GaAs/GaMnAs core-shell nanowires grown by molecular beam epitaxy. Nano Lett. 9, 3860- 3866 (2009). 
19. Siusys, A., Sadowski, J., et al. All wurtzite (In,Ga)As-(Ga,Mn)As core-shell nanowires. Nano Lett. 14, 4263-4272 (2014).

20. Sadowski, J. et al. Wurtzite (Ga,Mn)As nanowire shells with ferromagnetic properties. Nanoscale 9, 2129- 2137 (2017).

21. Liu, Yi. et al. Self-selective formation of ordered 1D and 2D GaBi structures on wurtzite GaAs nanowire surfaces. Nat. Commun. 12, 5990 (2021).

22. Laukkanen, P., Sadowski, J., \& Guina, M. Characterization of III-V surfaces with low energy electron diffraction and reflection high-energy-electron diffraction. in: Patane, A., \& Balkan, N.; (eds), Semiconductor Research, Springer Series in Materials Science 150, 121 (2012).

23. Kret, S., Kaleta, A., Bilska, M., Kurowska, B., Siusys, A., Dabrowski, J., Sadowski, J. FIB Method of Sectioning of III-V Core-multi-shell nanowires for analysis of core/shell interfaces by high resolution TEM. Acta Phys. Pol., A 131, 1332-1335 (2017).

24. Panciera, Federico et al. Phase selection in self-catalyzed GaAs nanowires. Nano Lett. 20, 1669-1675 (2020).

25. Jaggi, R. Struktur und eigenschaften der hochdruck-modifikation Bi II, Helv. Phys. Acta, 37, 618-619 (1964). 
26. Dubrovskii, V. G., Sibirev, N. V., Cirlin, G. E., Harmand, J. C., and Ustinov, V. M. Theoretical analysis of the vapor-liquid-solid mechanism of nanowire growth during molecular beam epitaxy. Phys. Rev. B 73, 021603 (2006).

27. Rudolph, Daniel et. al. Spontaneous alloy composition ordering in GaAs-AlGaAs coreshell nanowires. Nano Lett. 13, 1522-1527 (2013).

28. Mancini, L. et al. Three-dimensional nanoscale study of Al segregation and quantum dot formation in GaAs/AlGaAs core-shell nanowires. Appl. Phys. Lett. 105, 243106 (2014).

29. Wolf, Daniel et al. Three-dimensional composition and electric potential mapping of III-V core-multishell nanowires by correlative STEM and holographic tomography. Nano Lett. 18, 4777- 4784 (2018).

30. Leandro, L. et al. Wurtzite AlGaAs nanowires. Sci. Rep. 10, 735 (2020).

31. Zhang, Yunyan et al. Highly strained III-V-V coaxial nanowire quantum wells with strong carrier confinement. ACS Nano 13, 5931- 5938 (2019).

32. Reyes, Daniel F., Bastiman, Faebian., Hunter Chris, J., Sales, David L., Sanchez, Ana M., David, John, P., R., \& González, David. Bismuth incorporation and the role of ordering in GaAsBi/GaAs structures. Nanoscale Res. Lett. 9, 23 (2014). 


\section{Acknowledgements}

This work is supported in part by the National Science Centre, Poland through grants 2016/21/B/ST5/03411, 2017/25/N/ST5, 2018/31/B/ST3/03610; by the EAgLE international project (FP7-REGPOT-2013-1, No. 316014), and by the Foundation for Polish Science through the IRA Programme co-financed by EU within SG OP (Grant No. MAB/2017/1). The authors thank Dr. A Pietruczik from IP PAS, for deposition of Au on GaAs(111)B wafers.

\section{Author Contributions}

J.S. grew the samples and designed the project. J.S., A.K., S.Kret. A.M.S contributed to the writing of the manuscript. TW performed SEM characterization, S.Kret., A.K., A.M.S S. Krivyj did TEM characterization and interpreted TEM results; B.K. M.B. D.J, contributed to preparation of TEM specimens. J. Z. D. did XRD measurements implemented in the Supporting Information file. All authors approved the final version of the manuscript. 


\section{Supplementary Files}

This is a list of supplementary files associated with this preprint. Click to download.

- Supplementaryinformationfinal.pdf 